\title{
Antimicrobial resistance surveillance of doripenem in China
}

\author{
Yun Li, Yuan Lv, Feng Xue, Bo Zheng, Jian Liu and Jia Zhang
}

To investigate the antibacterial resistance to doripenem in China and to understand the distribution trends of resistant bacteria. All the clinical isolates were collected from hospitals and the susceptibility tests were performed using the agar dilution method recommended by the Clinical Laboratory Standards Institute (CLSI) central laboratory. The susceptibility of the isolates to antimicrobial agents was determined using the CLSI (2014) or European Committee on Antimicrobial Susceptibility Testing (EUCAST) (2013) guidelines. A total of 4047 pathogenic strains were isolated from 18 tertiary hospitals in 18 cities across China between July 2011 and June 2012. MIC results indicated that the vast majority of Enterobacteriaceae maintained high susceptibility to doripenem, with a lower resistance rate (1.9\%) than that observed for other drugs tested. In the case of nonfermenting Gram-negative isolates, the resistance rate of Pseudomonas aeruginosa was $16.2 \%$, which was less than that of imipenem and meropenem, and the Acinetobacter baumannii doripenem resistance rate was 67.4\%. Doripenem also showed good in vitro activity against other the bacteria tested. This study suggests that the gradual increase in carbapenem nonsusceptible Enterobacteriaceae should be monitored carefully alongside the increasing multidrug-resistant and extensively drug-resistant $A$. baumannii.

The Journal of Antibiotics (2015) 68, 496-500; doi:10.1038/ja.2015.25; published online 8 April 2015

\section{INTRODUCTION}

Carbapenems are a first choice treatment against serious bacterial infections due to their broad specificity and potent activity. ${ }^{1}$ Doripenem (S-4661) is a carbapenem developed by Shionogi and approved for marketing in Japan in September 2005. ${ }^{2}$ This drug has broad-spectrum activity against Gram-positive cocci and Gramnegative bacteria and anaerobes, and is stable against extendedspectrum $\beta$-lactamases, AmpC $\beta$-lactamases and dehydropeptidase-I. The post-antibiotic effect (PAE) of doripenem against Pseudomonas aeruginosa is $\sim 2 \mathrm{~h}$ in vitro, and MIC values are $2-4$-fold less than that observed for imipenem and meropenem. Pharmacokinetic parameters are similar to meropenem, with a bound-drug concentration in plasma of $8.9 \% .^{3,4}$ In October 2007, the US Food and Drug Administration (FDA) approved doripenem as a new drug to treat complicated urinary tract and intra-abdominal infections. ${ }^{5}$ The purpose of this study was to evaluate the in vitro activity of doripenem against a collection of pathogens isolated from tertiary hospitals in China.

\section{MATERIALS AND METHODS}

Bacterial strains

A total of 4047 strains were isolated from 18 tertiary hospitals in 18 cities across China between July 2011 and June 2012; Escherichia coli ATCC25922, E. coli ATCC35218, P. aeruginosa ATCC27853, Haemophilus influenzae ATCC49247, Staphylococcus aureus ATCC29213 and Streptococcus pneumoniae ATCC49619 was used as the control strain.
Antimicrobials

Doripenem and moxalactam were provided by Shionogi. Penicillin, oxacillin, ampicillin, piperacillin, meropenem, tazobactam, gentamicin and minocyclin were purchased from the National Institute for the Control of Pharmaceutical and Biological Products of China. Colistin and chloramphenicol were purchased from Sigma (Shanghai, China). Cefotaxime and cefoperazone were provided by China Nanjing Youke Biomedical Co. (Nanjing, China) Ceftazidime was provided by China Shenzhen Jiuxin Pharma Co., and cefepime was purchased from Bristol-Myers Squibb (Shanghai, China). Cefoxitin was provided by China Haikou Pharma Co, imipenem was purchased from Merck (Elkton, VA, USA), and sulbactam and Azithromycin were from Pfizer (Dalian, China). Levofloxacin was obtained from DaiichiSankyo Co, sulfamethoxazole and trimethoprim were from China Shandong Xinda Pharma Co, and vancomycin was purchased from Eli Lilly (Kobe, Japan).

\section{Susceptibility test}

MICs were performed using the agar dilution method recommended by the Clinical Laboratory Standards Institute (CLSI) central laboratory.

MIC assays

Susceptibility of isolates to antimicrobial agents was determined using CLSI (2014). The U.S. FDA susceptibility criteria of doripenem (in doripenem package insert) were used same time to Enterobacteriaceae and Acinetobacter baumannii because these differ from CLSI. 
Table 1 Antibacterial activity ( $\mu \mathrm{g} \mathrm{ml}^{-1}$ ) of antimicrobial agents against $E$. coli

\begin{tabular}{|c|c|c|c|c|c|c|c|c|c|c|c|c|}
\hline \multirow[b]{2}{*}{ Agents } & \multicolumn{4}{|c|}{ E. coli (600) } & \multicolumn{4}{|c|}{ ESBLs ${ }^{-}$E. coli (184) } & \multicolumn{4}{|c|}{$E S B L s^{+}$E. coli (416) } \\
\hline & $M I C_{50}$ & $M / C_{90}$ & $S \%$ & $R \%$ & $M I C_{50}$ & $M_{1} C_{90}$ & $S \%$ & $R \%$ & $M I C_{50}$ & $M I C_{90}$ & $S \%$ & $R \%$ \\
\hline Doripenem & 0.01 & 0.03 & 99.5 & 0.3 & 0.01 & 0.03 & 98.9 & 1.1 & 0.01 & 0.03 & 99.8 & 0.0 \\
\hline Doripenem $^{a}$ & - & - & 99.2 & 0.8 & - & - & 97.8 & 2.2 & - & - & 99.8 & 0.2 \\
\hline Imepenem & 0.13 & 0.25 & 98.8 & 0.3 & 0.13 & 0.25 & 97.8 & 1.1 & 0.13 & 0.25 & 99.3 & 0.0 \\
\hline Meropenem & 0.01 & 0.06 & 99.3 & 0.3 & 0.01 & 0.03 & 97.8 & 1.1 & 0.01 & 0.06 & 100.0 & 0.0 \\
\hline Ampicillin & $>256$ & $>256$ & 6.0 & 93.8 & $>256$ & $>256$ & 19.6 & 79.9 & $>256$ & $>256$ & 0.0 & 100.0 \\
\hline Cefotaxime & 32 & $>256$ & 26.7 & 73.0 & 0.06 & 16 & 86.4 & 13.6 & 64 & $>256$ & 0.2 & 99.3 \\
\hline Ceftazidime & 2 & 64 & 55.7 & 36.8 & 0.13 & 32 & 85.3 & 14.1 & 8 & 64 & 42.5 & 46.9 \\
\hline Cefepime & 4 & 32 & 41.7 & 27.5 & 0.03 & 1 & 91.8 & 5.4 & 8 & 64 & 19.5 & 37.3 \\
\hline Moxalactam & 0.25 & 2 & 95.7 & 1.3 & 0.13 & 4 & 92.4 & 2.2 & 0.5 & 2 & 97.1 & 1.0 \\
\hline $\mathrm{TZP}^{\mathrm{b}}$ & 8 & 32 & 85.7 & 3.0 & 4 & 32 & 87.5 & 4.3 & 8 & 32 & 84.9 & 2.4 \\
\hline $\mathrm{CSL}^{\mathrm{C}}$ & 8 & 32 & 80.7 & 9.0 & 0.5 & 16 & 92.9 & 3.3 & 16 & 64 & 75.2 & 11.5 \\
\hline Gentamicin & 32 & 128 & 38.5 & 59.8 & 16 & 128 & 46.2 & 52.7 & 32 & 128 & 35.1 & 63.0 \\
\hline Minocyclin & 4 & 32 & 63.2 & 26.0 & 2 & 32 & 67.9 & 21.2 & 4 & 32 & 61.1 & 28.1 \\
\hline Levofloxacin & 8 & 32 & 34.2 & 58.0 & 1 & 16 & 52.2 & 37.0 & 8 & 32 & 26.2 & 67.3 \\
\hline
\end{tabular}

Abbreviatons: $\mathrm{R}$, resistance; $\mathrm{S}$, susceptibility.

aAccording to the US FDA criteria ( $\mathrm{S}: \leqslant 0.5 \mu \mathrm{g} \mathrm{ml}^{-1}$; nonsusceptible: $>0.5 \mu \mathrm{g} \mathrm{ml}^{-1}$ ).

bPiperacillin/tazobactam $(8: 1)$.

cefoperazone/sulbactam $(2: 1)$. The breakpoint of cefoperazone was used for CSL $\left(\mathrm{S}: \leqslant 16 \mu \mathrm{g} \mathrm{ml}^{-1} ; \mathrm{R}: \geqslant 64 \mu \mathrm{g} \mathrm{ml}{ }^{-1}\right)$.

Table 2 Antibacterial activity ( $\mu \mathrm{g} \mathrm{ml}^{-1}$ ) of antimicrobial agents against $K$. pneumonia

\begin{tabular}{|c|c|c|c|c|c|c|c|c|c|c|c|c|}
\hline \multirow[b]{2}{*}{ Agents } & \multicolumn{4}{|c|}{ K. pneumoniae (500) } & \multicolumn{4}{|c|}{ ESBLs ${ }^{-}$K. pneumoniae (279) } & \multicolumn{4}{|c|}{ ESBLs ${ }^{+}$K. pneumoniae (221) } \\
\hline & $M I C_{50}$ & $M_{1} C_{90}$ & $S \%$ & $R \%$ & $M I C_{50}$ & $M I C_{90}$ & $S \%$ & $R \%$ & $M I C_{50}$ & $M I C_{90}$ & $S \%$ & $R \%$ \\
\hline Doripenem & 0.03 & 0.06 & 95.8 & 3.4 & 0.03 & 0.13 & 92.5 & 6.1 & 0.03 & 0.06 & 100.0 & 0.0 \\
\hline Doripenema & - & - & 95.2 & 4.8 & - & - & 91.8 & 8.2 & - & - & 99.5 & 0.5 \\
\hline Imepenem & 0.13 & 0.25 & 95.8 & 3.6 & 0.25 & 0.5 & 92.8 & 6.5 & 0.13 & 0.25 & 99.5 & 0.0 \\
\hline Meropenem & 0.03 & 0.06 & 95.6 & 3.6 & 0.03 & 0.25 & 92.1 & 6.5 & 0.03 & 0.06 & 100.0 & 0.0 \\
\hline Cefotaxime & 4 & 256 & 48.0 & 50.2 & 0.03 & 32 & 84.9 & 12.9 & 64 & 256 & 1.4 & 97.3 \\
\hline Ceftazidime & 1 & 128 & 65.4 & 28.8 & 0.13 & 32 & 85.7 & 12.2 & 8 & 128 & 39.8 & 49.8 \\
\hline Cefepime & 0.5 & 32 & 62.4 & 23.4 & 0.03 & 4 & 89.6 & 9.0 & 8 & 64 & 28.1 & 41.6 \\
\hline Moxalactam & 0.25 & 4 & 93.8 & 4.2 & 0.13 & 8 & 90.0 & 6.8 & 0.5 & 2 & 98.6 & 0.9 \\
\hline $\mathrm{TZPb}$ & 8 & 64 & 77.6 & 8.6 & 4 & 128 & 85.7 & 11.1 & 16 & 64 & 67.4 & 5.4 \\
\hline $\mathrm{CSL}^{\mathrm{C}}$ & 4 & 64 & 74.4 & 12.6 & 0.25 & 64 & 86.7 & 10.8 & 16 & 64 & 58.8 & 14.9 \\
\hline Gentamicin & 0.5 & 128 & 62.4 & 36.6 & 0.5 & 32 & 86.7 & 12.2 & 32 & $>256$ & 31.7 & 67.4 \\
\hline Minocyclin & 2 & 32 & 67.0 & 25.2 & 2 & 16 & 82.4 & 10.8 & 8 & 64 & 47.5 & 43.4 \\
\hline Levofloxacin & 0.25 & 32 & 74.2 & 23.4 & 0.06 & 8 & 87.5 & 11.1 & 1 & 64 & 57.5 & 38.9 \\
\hline
\end{tabular}

Abbreviatons: $\mathrm{R}$, resistance; $\mathrm{S}$, susceptibility.

${ }^{a}$ According to the US FDA criteria ( $\mathrm{S}: \leqslant 0.5 \mu \mathrm{g} \mathrm{ml}^{-1}$; nonsusceptible: $>0.5 \mu \mathrm{g} \mathrm{ml}^{-1}$ ).

bPiperacillin/tazobactam $(8: 1)$.

'Cefoperazone/sulbactam (2:1). The breakpoint of cefoperazone was used for CSL (S: $\left.\leqslant 16 \mu \mathrm{gl}^{-1} ; \mathrm{R}: \geqslant 64 \mu \mathrm{gl}^{-1}\right)$.

\section{RESULTS}

Antibacterial activity of doripenem and other antimicrobial agents against Enterobacteriaceae

The detection rates of extended-spectrum beta-lactamase (ESBL)producing E. coli and Klebsiella pneumoniae were $69.3 \%$ and $44.2 \%$, respectively (Tables 1 and 2). Doripenem showed potent in vitro activity against Enterobacteriaceae. The susceptibility for doripenem against all Enterobacteriaceae isolates was $>95 \%\left(\mathrm{MIC}_{90} \leqslant 0.25 \mu \mathrm{g} \mathrm{ml}^{-1}\right.$ for different genera). Carbapenems are important Enterobacteriaceae infection treatments, and this was reflected in the resistance rates for doripenem, meropenem and imipenem of $1.9 \%, 2.0 \%$ and $2.3 \%$, respectively. The antimicrobial activity of doripenem was similar to meropenem, and slightly higher than imipenem (Tables 1-3). In addition, moxalactam, piperacillin/tazobactam and cefoperazone/ sulbactam exhibited a strong activity against Enterobacteriaceae isolates. Carbapenem followed by moxalactam showed the highest antibacterial activity against ESBL-positive E. coli and $K$. pneumoniae.

Antibacterial activity of doripenem and other antimicrobial agents against non-fermenting Gram-negative bacteria

Rates of resistance for most antimicrobial agents against $P$. aeruginosa ranged from 10 to $30 \%$ (Tables 4 and 5), and was $16.2 \%$ for doripenem, lower than for meropenem and imipenem. The susceptibility of imipenem-susceptible and imipenem-resistant $P$. aeruginosa to doripenem was 99.5 and $39.9 \%$, respectively, and partial cross-resistance occurred in some cases, suggesting doripenem remained effective against some imipenem-resistant $P$. aeruginosa. Additionally, ceftazidime and cefepime were also effective against 
Table 3 Antibacterial activity $\left(\mu \mathrm{g} \mathrm{ml}^{-1}\right.$ ) of antimicrobial agents against other Enterobacteriaceae

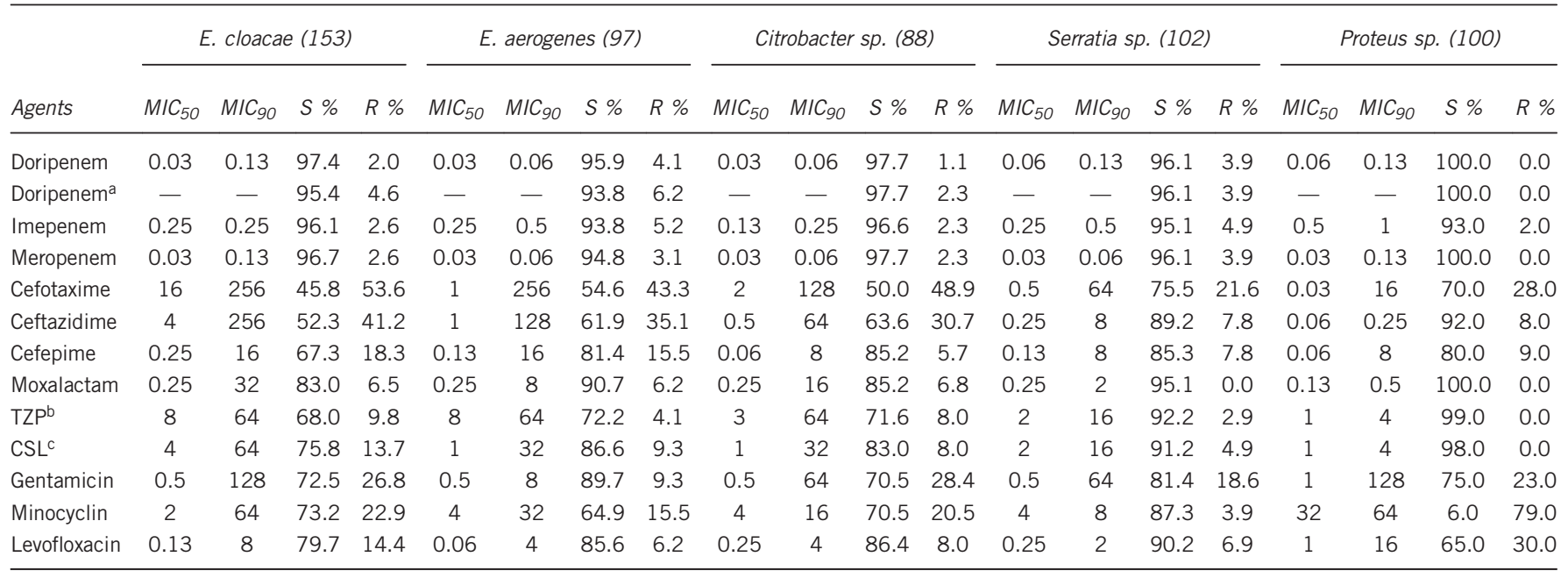

Abbreviatons: R, resistance; S, susceptibility.

according US FDA criteria ( $\mathrm{S}: \leqslant 0.5 \mu \mathrm{g} \mathrm{ml}^{-1}$; nonsusceptible: $>0.5 \mu \mathrm{g} \mathrm{ml}^{-1}$ ),

bPiperacillin/tazobactam $(8: 1)$.

${ }^{c}$ Cefoperazone/sulbactam $(2: 1)$. The breakpoint of cefoperazone was used for CSL (S: $\leqslant 16 \mu \mathrm{gl}^{-1} ; \mathrm{R}: \geqslant 64 \mu \mathrm{g} \mathrm{ml} \mathrm{l}^{-1}$ ).

Table 4 Antibacterial activity $\left(\mu \mathrm{g} \mathrm{ml}{ }^{-1}\right.$ ) of antimicrobial agents against $P$. aeruginosa

\begin{tabular}{|c|c|c|c|c|c|c|c|c|c|c|c|c|}
\hline \multirow[b]{2}{*}{ Agents } & \multicolumn{4}{|c|}{$P$. aeruginosa (610) } & \multicolumn{4}{|c|}{ IMP-S P. aeruginosa $(367)^{\circledR}$} & \multicolumn{4}{|c|}{ IMP-NS P. aeruginosa $(243)^{b}$} \\
\hline & $M I C_{50}$ & $M I C_{90}$ & $S \%$ & $R \%$ & $M I C_{50}$ & $M I C_{90}$ & $S \%$ & $R \%$ & $M I C_{50}$ & $M I C_{90}$ & $S \%$ & $R \%$ \\
\hline Doripenem & 0.5 & 8 & 73.8 & 16.2 & 0.25 & 1 & 99.5 & 0.5 & 4 & 32 & 35.0 & 39.9 \\
\hline Imepenem & 2 & 16 & 60.2 & 31.3 & 2 & 2 & 100.0 & 0.0 & 16 & 32 & 0.0 & 78.6 \\
\hline Meropenem & 0.5 & 16 & 68.2 & 24.4 & 0.25 & 1 & 97.5 & 0.8 & 8 & 32 & 23.9 & 60.1 \\
\hline Ceftazidime & 2 & 64 & 75.9 & 18.5 & 2 & 16 & 87.7 & 8.4 & 8 & $>256$ & 58.0 & 33.7 \\
\hline Cefepime & 2 & 32 & 76.7 & 12.0 & 2 & 8 & 91.6 & 3.5 & 8 & 128 & 54.3 & 24.7 \\
\hline Moxalactamc & 32 & 128 & 17.0 & 36.7 & 16 & 64 & 21.0 & 21.8 & 64 & 256 & 11.1 & 59.3 \\
\hline TZP & 8 & 128 & 68.9 & 10.3 & 4 & 64 & 82.6 & 4.4 & 32 & 128 & 48.1 & 19.3 \\
\hline $\mathrm{CSL}^{\mathrm{e}}$ & 8 & 128 & 66.7 & 21.5 & 8 & 32 & 82.6 & 7.6 & 32 & 128 & 42.8 & 42.4 \\
\hline Gentamicin & 2 & $>256$ & 72.1 & 25.1 & 2 & $>256$ & 83.1 & 14.2 & 4 & $>256$ & 55.6 & 41.6 \\
\hline Levofloxacin & 1 & 16 & 65.7 & 26.1 & 0.5 & 8 & 82.3 & 12.5 & 4 & 64 & 40.7 & 46.5 \\
\hline
\end{tabular}

aIMP-S: Imipenem-susceptibility.

bIMP-NS: Imipenem -nonsusceptibility.

'The breakpoint of moxalactam against Enterobacteriaceae was used here $\left(\mathrm{S}: \leqslant 8 \mu \mathrm{g} \mathrm{ml}-1\right.$; $\left.\mathrm{R}: \geqslant 64 \mu \mathrm{g} \mathrm{ml}^{-1}\right)$.

dPiperacillin/tazobactam $(8: 1)$.

e Cefoperazone/sulbactam (2:1). The breakpoint of cefoperazone against Enterobacteriaceae was used for CSL (S: $\left.\leqslant 16 \mu \mathrm{gl}^{-1} ; \mathrm{R}: \geqslant 64 \mu \mathrm{g} \mathrm{ml}{ }^{-1}\right)$.

imipenem-susceptible $P$. aeruginosa, but moxalactam was not very active against $P$. aeruginosa.

The resistance rates for $A$. baumannii against most antimicrobial agents ranged from 60 to $80 \%$, and the resistance to doripenem was $67.4 \%$, which was comparable to meropenem and imipenem. The rates of susceptibility and resistance of imipenem-susceptible A. baumannii to doripenem were $95.9 \%$ and $0.5 \%$, respectively. Neither doripenem nor meropenem were active against imipenemnonsusceptible strains. The susceptibility to colistin was $96.6 \%$, which was the most active antibacterial agent against this species.

Antibacterial activity of doripenem and other antimicrobial agents against Gram-positive bacteria

The susceptibility of methicillin-resistant $S$. aureus and methicillinresistant S. epidermidis to doripenem were $45.4 \%$ and $81.6 \%$, respectively (Tables 6 and 7). The revised CLSI (2014) guidelines eliminated breakpoints (interpretive criteria) for all $\beta$-lactams except oxacillin, cefoxitin and penicillin, and included breakpoints for ceftaroline (a cephalosporin with anti-methicillin-resistant S. aureus activity). We did not calculate the rates of susceptibility or resistance for antibacterial agents for which the breakpoint data were not included in these guidelines.

The $\mathrm{MIC}_{90}$ values for doripenem against methicillin-sensitive Staphylococcus sp. were $<0.5 \mu \mathrm{g} \mathrm{ml}^{-1}$, which demonstrated the effectiveness of doripenem against these strains. Similar findings were observed for imipenem and meropenem. All other agents except penicillin and azithromycin showed a strong activity against methicillin-sensitive Staphylococcus sp., and vancomycin resistance was not observed in staphylococci. No $\beta$-lactam antimicrobial drugs, even when active in vitro, were active against methicillin-resistant $S$. aureus in clinical treatments, suggesting that the isolated strains could be classified as resistant. 
Table 5 Antibacterial activity ( $\mu \mathrm{g} \mathrm{ml}^{-1}$ ) of antimicrobial agents against $A$. baumannii

\begin{tabular}{|c|c|c|c|c|c|c|c|c|c|c|c|c|}
\hline & \multicolumn{4}{|c|}{ A. baumannii (594) } & \multicolumn{4}{|c|}{ IMP-S A. baumannii (194)a } & \multicolumn{4}{|c|}{ IMP-NS A. baumannii $(400)^{\mathrm{b}}$} \\
\hline & $M / C_{50}$ & $M / C_{90}$ & $S \%$ & $R \%$ & $M / C_{50}$ & $M / C_{90}$ & $S \%$ & $R \%$ & $M / C_{50}$ & $M / C_{90}$ & $S \%$ & $R \%$ \\
\hline Doripenem & 32 & 64 & 31.3 & 67.4 & 0.25 & 2 & 95.9 & 0.5 & 32 & 64 & 0.0 & 99.7 \\
\hline Doripenem $^{\mathrm{C}}$ & - & - & 27.8 & 72.2 & - & - & 85.1 & 14.9 & - & - & 0.0 & 100.0 \\
\hline Imepenem & 32 & 64 & 32.7 & 66.3 & 0.25 & 2 & 100.0 & 0.0 & 32 & 64 & 0.0 & 98.5 \\
\hline Meropenem & 32 & 128 & 29.6 & 68.0 & 0.5 & 4 & 90.7 & 2.6 & 64 & 128 & 0.0 & 99.7 \\
\hline $\mathrm{SAM}^{\mathrm{d}}$ & 64 & 128 & 22.6 & 73.6 & 2 & 32 & 69.1 & 24.7 & 64 & 128 & 0.0 & 97.2 \\
\hline Cefotaxime & $>256$ & $>256$ & 12.8 & 75.1 & 16 & $>256$ & 38.1 & 28.9 & $>256$ & $>256$ & 0.5 & 97.5 \\
\hline Ceftazidime & 64 & $>256$ & 25.3 & 74.1 & 4 & 128 & 71.1 & 27.9 & 128 & $>256$ & 3.0 & 96.5 \\
\hline Cefepime & 32 & 256 & 25.4 & 62.8 & 2 & 32 & 75.3 & 11.8 & 64 & 256 & 1.3 & 87.5 \\
\hline Moxalactame & 128 & 256 & 1.5 & 82.7 & 32 & 128 & 4.6 & 48.5 & 128 & 256 & 0.0 & 99.2 \\
\hline $\mathrm{TZP}^{f}$ & 128 & $>256$ & 22.6 & 67.5 & 16 & 64 & 68.6 & 7.2 & 256 & $>256$ & 0.3 & 96.7 \\
\hline $\operatorname{CSL}^{\mathrm{g}}$ & 32 & 128 & 28.6 & 49.2 & 2 & 32 & 76.8 & 9.3 & 64 & 128 & 5.3 & 68.5 \\
\hline Gentamicin & $>256$ & $>256$ & 24.2 & 75.4 & 1 & $>256$ & 66.0 & 33.5 & $>256$ & $>256$ & 4.0 & 95.7 \\
\hline Minocyclin & 4 & 16 & 58.4 & 22.7 & 0.25 & 8 & 87.6 & 7.2 & 8 & 32 & 44.3 & 30.2 \\
\hline Levofloxacin & 8 & 16 & 25.3 & 54.7 & 0.13 & 8 & 71.6 & 19.6 & 8 & 16 & 2.8 & 71.7 \\
\hline Colistin & 1 & 2 & 96.6 & 3.4 & 1 & 2 & 95.9 & 4.1 & 1 & 1 & 97.0 & 3.0 \\
\hline
\end{tabular}

aIMP-S: Imipenem-susceptibility.

bIMP-NS: Imipenem -nonsusceptibility.

According to the US FDA criteria (S: $\leqslant 1 \mu \mathrm{g} \mathrm{ml}^{-1}$; Nonsusceptible: $>1 \mu \mathrm{g} \mathrm{ml}-1$ ).

dAmpicillin/sulbactam (2:1).

eThe breakpoint of moxalactam against Enterobacteriaceae was used here $\left(\mathrm{S}: \leqslant 8 \mu \mathrm{g} \mathrm{ml}-1 ; \mathrm{R}: \geqslant 64 \mu \mathrm{g} \mathrm{ml}^{-1}\right)$.

fPiperacillin/tazobactam $(8: 1)$.

gCefoperazone/sulbactam (2:1). The breakpoint of cefoperazone against Enterobacteriaceae was used for CSL (S: $\leqslant 16 \mathrm{mg} / \mathrm{l} ; \mathrm{R}: \geqslant 64 \mathrm{mg} / \mathrm{l})$.

Table 6 Antibacterial activity $\left(\mu \mathrm{g} \mathrm{ml}^{-1}\right.$ ) of antimicrobial agents against $S$. aureus and $S$. epidermidis

\begin{tabular}{|c|c|c|c|c|c|c|c|c|c|c|c|c|c|c|c|c|}
\hline \multirow[b]{2}{*}{ Agents } & \multicolumn{4}{|c|}{ S. aureus (601) } & \multicolumn{4}{|c|}{ MSSA (328) } & \multicolumn{4}{|c|}{ S. epidermidis (98) } & \multicolumn{4}{|c|}{ MSSE (18) } \\
\hline & $M I C_{50}$ & $M I C_{90}$ & $S \%$ & $R \%$ & $M / C_{50}$ & $M I C_{90}$ & $S \%$ & $R \%$ & $M I C_{50}$ & $M I C_{90}$ & $S \%$ & $R \%$ & $M I C_{50}$ & $M I C_{90}$ & $S \%$ & $R \%$ \\
\hline Doripenem & 0.06 & 32 & - & - & 0.03 & 0.06 & - & - & 0.25 & 2 & - & - & 0.03 & 0.5 & - & - \\
\hline Imepenem & 0.03 & 64 & - & - & 0.03 & 0.03 & - & - & 0.13 & 0.5 & - & - & 0.01 & 0.25 & - & - \\
\hline Meropenem & 0.25 & 32 & - & - & 0.13 & 0.25 & - & - & 0.5 & 2 & - & - & 0.06 & 2 & - & - \\
\hline Penicillin & 1 & 64 & 9.5 & 90.5 & 0.5 & 1 & 16.2 & 83.8 & 1 & 8 & 14.3 & 85.7 & 0.13 & 0.5 & 55.6 & 44.4 \\
\hline Oxacillin & 0.5 & 256 & 57.4 & 42.6 & 0.25 & 0.5 & 100.0 & 0.0 & 1 & 32 & 18.4 & 81.6 & 0.13 & 0.25 & 100.0 & 0.0 \\
\hline Cefoxitin & 4 & 256 & 55.1 & 44.9 & 2 & 4 & 100.0 & 0.0 & 8 & 32 & - & - & 2 & 4 & - & - \\
\hline Moxalactam & 16 & $>256$ & - & - & 8 & 16 & - & - & 32 & 256 & - & - & 16 & 256 & - & - \\
\hline TZPa & 4 & 128 & - & - & 2 & 4 & - & - & 2 & 4 & - & - & 0.5 & 2 & - & - \\
\hline Gentamicin & 0.25 & 64 & 53.6 & 41.9 & 0.25 & 16 & 79.9 & 14.3 & 0.25 & 32 & 70.4 & 18.4 & 0.13 & 16 & 88.9 & 11.1 \\
\hline Azithromycin & $>256$ & $>256$ & 23.8 & 74.5 & $>256$ & $>256$ & 32.0 & 68.0 & 64 & $>256$ & 17.3 & 81.6 & 64 & $>256$ & 38.9 & 61.1 \\
\hline Minocyclin & 0.06 & 2 & 97.8 & 0.7 & 0.06 & 0.13 & 100.0 & 0.0 & 0.13 & 0.5 & 100.0 & 0.0 & 0.06 & 0.25 & 100.0 & 0.0 \\
\hline Levofloxacin & 0.25 & 64 & 56.2 & 43.1 & 0.25 & 4 & 87.8 & 11.3 & 2 & 8 & 45.9 & 38.8 & 0.13 & 4 & 72.2 & 22.2 \\
\hline Vancomycin & 0.5 & 1 & 100.0 & 0.0 & 0.5 & 1 & 100.0 & 0.0 & 1 & 2 & 100.0 & 0.0 & 1 & 1 & 100.0 & 0.0 \\
\hline
\end{tabular}

-: No breakpoint.

apiperacillin/tazobactam $(8: 1)$

The rates of resistance and intermediate resistance of penicillin against S. pneumoniae strains (not including meningitis isolates) were $1.5 \%$ and $7.8 \%$, respectively. Susceptibility to doripenem was $91.9 \%$, which was significantly higher than imipenem and meropenem. Penicillin, levofloxacin and vancomycin also showed good activity against S. pneumoniae. Streptococcus pyogenes isolates were also susceptible to most antimicrobial agents tested except azithromycin (resistance rate $=92.3 \%$ ), and the susceptibility for doripenem was $100 \%\left(\mathrm{MIC} \leqslant 0.06 \mu \mathrm{g} \mathrm{ml}^{-1}\right.$ ). Other $\beta$-hemolytic isolates were also susceptible to most antimicrobial agents, and the susceptibility to doripenem equaled to meropenem in $98.1 \%$ of cases. The susceptibility of viridans isolates to doripenem was $92.7 \%$, which was higher than that for all other agents tested except vancomycin.

\section{DISCUSSION}

The results of this extensive study confirmed that doripenem maintained strong antibacterial activity against Enterobacteriaceae, with a resistance of just $1.9 \%$, which was lower than all other carbapenems tested. The mean resistance rate of $K$. pneumoniae isolates toward carbapenems ranged from 3.4 to $3.6 \%$. These strains are known to produce carbapenemases or other $\beta$-lactamases that are present in ESBL-negative groups but are not inhibited by clavulanic acid. Resistance to carbapenems was therefore higher in ESBL-negative bacilli than in ESBL-producing strains. It is worth noting that increasing resistance against carbapenems has emerged in K. pneumoniae. ${ }^{6}$ Compared with imipenem and meropenem, doripenem (resistance $=16.2 \%$ ) was more active against $P$. aeruginosa. 
Table 7 Antibacterial activity $\left(\mu \mathrm{g} \mathrm{ml}^{-1}\right.$ ) of antimicrobial agents against Streptococcus sp.

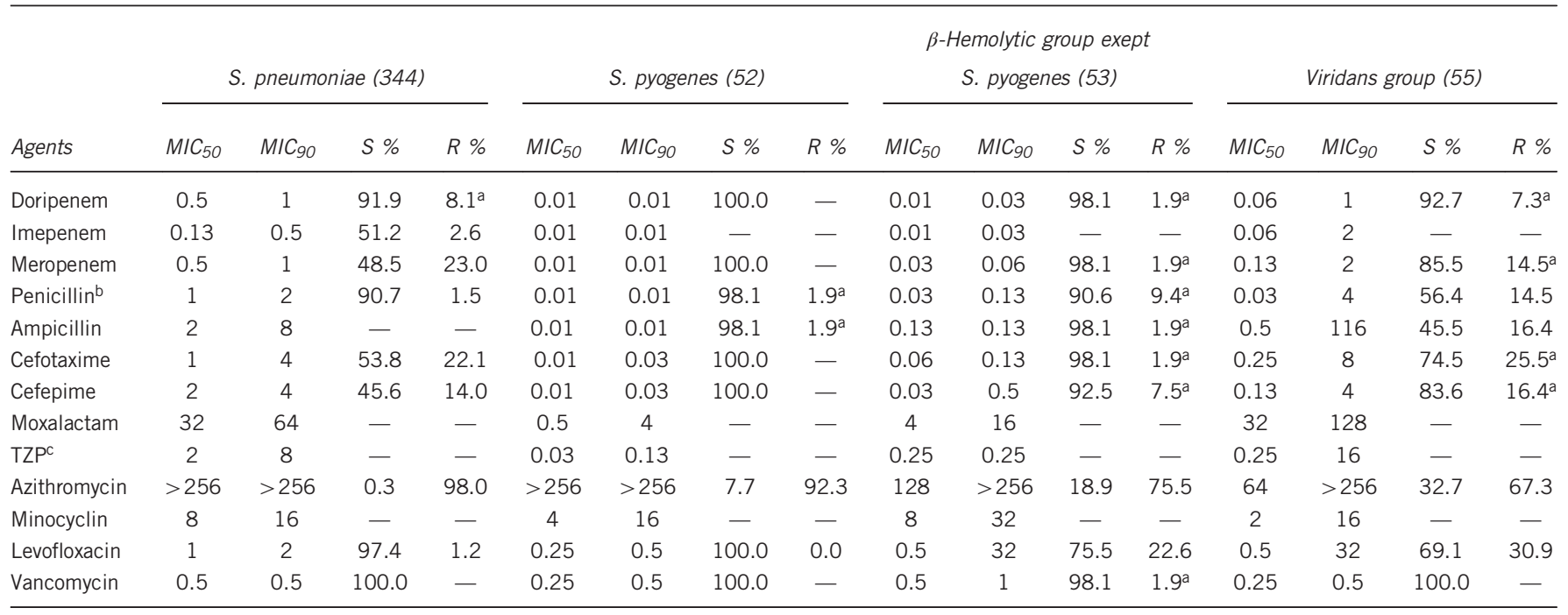

-: No breakpoint.

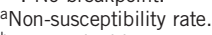

bNonmeningitis breakpoint.

cPiperacillin/tazobactam (8:1).

Carbapenem-resistance has also increased in A. baumannii, from $1 \%$ in 2000-2001 to $>60 \%$ more recently. ${ }^{6-11}$ Multidrug-resistant A. baumannii is a particular concern, and can account for $80.3 \%$ of strains. ${ }^{11}$ The doripenem activity against Enterobacteriaceae ( $\mathrm{MIC}_{90}$ $\leqslant 0.25 \mu \mathrm{g} \mathrm{ml}^{-1}$ for different genus) and $P$. aeruginosa (MIC 90 $8 \mu \mathrm{g} \mathrm{ml}^{-1}$ ) determined in this study are in agreement with previously published international reports $\left(\mathrm{MIC}_{90}=0.06-0.5 \mu \mathrm{g} \mathrm{ml}^{-1}\right.$ for Enterobacteriaceae and $\mathrm{MIC}_{90}=1-16 \mu \mathrm{g} \mathrm{ml}^{-1}$ for $P$. aeruginosa. ${ }^{12,13}$ However, our data for A. baumannii, differed from the published Surveillance of Multicentre Antimicrobial Resistance in Taiwan (SMART) surveillance data. ${ }^{12} \mathrm{~A}$. baumannii susceptibility to doripenem was $31.3 \%$ in this study, which was much lower than the SMART surveillance figure of $79 \%$. Similarly, $\mathrm{MIC}_{90}$ values were 64 and $16 \mu \mathrm{g} \mathrm{ml}^{-1}$ in the current study and SMART surveillance report. ${ }^{12}$ These differences may be due to different criteria studied, since we used $\leqslant 2 \mu \mathrm{g} \mathrm{ml}^{-1}$ as the susceptible breakpoint for doripenem against A. baumannii based on the CLSI 2014 report, whereas the SMART surveillance report used $\leqslant 4 \mu \mathrm{g} \mathrm{ml}^{-1}$. In addition, specimen collection times were different: we collected strains between July 2011 and June 2012, whereas the SMART surveillance collected the specimens between September and November 2005. Differences in the geographical area where organisms were collected is another potential source of inconsistency: our strains were collected from mainland China, whereas the SMART surveillance samples were isolated in Taiwan. These factors may all contribute to the differences observed. As stated above, carbapenem-resistant $A$. baumannii have increased dramatically during the past decade, and therefore have a higher MIC than in the past. Using a lower susceptibility breakpoint will therefore result in a lower rate of susceptibility.

\section{ACKNOWLEDGEMENTS}

The following hospitals contributed to this study: (1) the Institute of Clinical Pharmacology, Peking University First Hospital, Beijing; (2) the Beijing Hospital, Beijing; (3) the Ji Lin University Second Hospital, Changchun; (4) the Tianjin Medical University General Hospital, Tianjin; (5) the Second Hospital of Hebei Medical University, Shijiazhuang; (6) the First Affiliated Hospital with Nanjing Medical University, Nanjing; (7) the Zhongshan Hospital Fudan
University, Shanghai; (8) Sir Run Run Shaw Hospital, School of Medicine, Zhejiang University, Hangzhou; (9) Guangzhou Women and Children's Medical Center, Guangzhou; (10) Renmin Hospital of Wuhan University, Wuhan; (11) Xiangya Hospital Central-South University, Changsha; (12) Kunming First People's Hospital, Kunming; (13) the Affiliated Hospital of Guiyang Medical College, Guiyang; (14 ) Southwest Hospital, Third Military Medical University, Chongqing; (15) Xijing Hospital, Forth Military Medical University, Xi'an; (16) Jinan Central Hospital Affiliated to Shandong University, Jinan; (17) the Lanzhou University Second Hospital, Lanzhou; (18) the First Teaching Hospital of Xinjiang Medical University, Wu lu mu qi; (19) the Hanzhong Center Hospital, Hanzhong.

1 Tsuji, M, Ishii, Y \& Ohno, A. In vitro and in vivo antibacterial activities of S-4661, a new carbapenem. Antimicrob. Agents Chemother. 42, 94-99 (1998).

2 Zheng, X. X., Hou, G. J. \& Lin, Z. M. Pharmacological characteristics and clinical application of doripenem. Evaluation and Analysis of Drug-use in Hospital of China 11, 195-197 (2011).

3 Jones, R. N., Huynh, H. K. \& Biedenbach, D. J. Activities of doripenem (S-4661) against drug-resistant clinical pathogens. Antimicrob. Agents Chemother. 48, 3136-3140 (2004).

4 Sahm, D. In vitro activity of doripenem. Clin. Infect. Dis. 49, S11-S16 (2009).

5 Marti, S., Sanchez-Cespedes, J., Alba, V. \& Vila, J. In vitro activity of doripenem against Acinetobacter baumannii clinical isolates. Inter. J. Antimicrob. Agents 33, 181-182 (2009).

$6 \mathrm{Li}, \mathrm{Y}$. et al. Anticicrobial susceptibility surveillance in China: a MOHNARIN program report (2009-2010). Clin. J. Lab. Med. 35, 67-87 (2012).

$7 \mathrm{Li}$, J.T., Li, Y. \& Wang, J. Surveillance on drug resistance of gram-negative bacilli isolated from hospital acquired infections and community acquired infections (2000-2001). Natl Med. J. China 83, 1035-1045 (2003).

8 Li, J.T., Li, Y. \& Qi, H. M. 2002-2003 bacterial resistance surveillance study on gram-negative bacilli in China. Chin. J. Lab. Med. 28, 19-29 (2005).

9 Li, Y., Xiao, Y. H. \& Wang, J. Surveillance of gram-negative bacterial resistance: data from Ministry of Health P. R. China National Antibacterial Resistance Investigation Net in 2004-2005. Chin. J. Infect. Dis. 26, 202-210 (2008).

$10 \mathrm{Li}, \mathrm{Y}$. et al. Antimicrobial susceptibility surveillance in China: a MOHNARIN program 2007-2008 report. Chin. J. Clin. Pharmacol. 27, 323-334 (2011).

$11 \mathrm{Li}, \mathrm{Y}$. et al. Antimicrobial susceptibility surveillance of gram-negative bacteria from MOHNARIN 2011-2012. Chin. J. Clin. Pharmacol. 30, 260-277 (2014).

12 Jean, S. S. et al. In vitro activities of doripenem and other carbapenems against clinically important bacteria isolated in intensive care units: nationwide data from SMART Programme. Eur. J. Clin. Microbiol. Infect. Dis. 29, 471-475 (2010).

13 Nordmann, P. et al. Comparative activity of carbapenem testing: the COMPACT study. J. Antimicrob. Chemother. 66, 1070-1078 (2011). 